\title{
A QUASI-EXPERIMENTAL STUDY TO ASSESS THE EFFECTIVENESS OF GARLIC SCENTED MUSTARD OIL FOOT MASSAGE ON FATIGUE AFTER CHILDBIRTH AMONG POSTNATAL MOTHERS IN SELECTED HOSPITALS OF DISTRICT MOHALI, PUNJAB.
}

\author{
Ms. Gazala Khursheed ${ }^{1}$, Mrs. Shivani Sharma ${ }^{2}$
}

\author{
${ }^{1}$ M.Sc. Nursing (Obstetrics and Gynaecological Nursing) Mata Sahib Kaur College of Nursing Balongi, Mohali, Punjab \\ ${ }^{2}$ Professor, Mata Sahib Kaur College of Nursing Balongi, Mohali, Punjab \\ Corresponding email: :- : -ayatwani66@gmail.com, sharmashivani@yahoo.ca
}

\begin{abstract}
:
Introduction :Pregnancy and childbirth are normal life events and involve mixed emotions, where the mother has a fear about childbirth process and also filled with happiness of having a baby. Fatigue diminishes mother's ability to concentrate on children and increases parenting stress which damages her daily functioning, communication with others and mother-infant relationships.

Material \& Method : A quantitative research approach with quasi-experimental non randomized control group research design was used. Study was conducted in selected hospitals of district Mohali, Punjab. 80 primiparous postnatal mothers were selected by using non-probability purposive sampling technique. Fatigue was assessed by using self-structured postnatal fatigue assessment scale and visual analogue fatigue scale.

Result: The results according to self-structured postnatal fatigue assessment scale showed that in experimental group, majority of postnatal mothers $(80 \%)$ had mild fatigue, $20 \%$ had moderate fatigue and none of them had either severe fatigue or no fatigue. According to visual analogue fatigue scale it showed that in the experimental group, majority of postnatal mothers $(77.5 \%$ ) had mild fatigue and $22.5 \%$ had moderate fatigue but none of them had either severe fatigue or no fatigue.

Conclusion: The study concluded that foot massage with garlic scented mustered oil was found to be very effective in reducing fatigue after childbirth among postnatal mothers.

Keywords: Garlic scented mustard oil foot massage, fatigue, childbirth, primparous mothers.
\end{abstract}

\section{INTRODUCTION}

The postpartum period, also called as postnatal or puerperal period is the period which begins immediately after birth of the baby and extends up to six weeks. During this period, the women's body and hormone level return to pre-pregnant state.

Postnatal mothers face difficult time after giving birth and have unmet needs for support in that time period. The mothers feel fatigue from childbirth process, breastfeeding and infant care early in their postnatal period. $^{2}$

Fatigue diminishes mother's ability to concentrate on children and increases parenting stress which damages her daily functioning, communication with others and mother-infant relationships. Infant's irregular sleep patterns and frequent feeds are typical in the early postpartum period and may contribute to maternal fatigue.
Massage is one of the most recommended types of alternative therapies to treat the symptoms of fatigue. Massage therapy has been shown to increase circulation, reduce stress, pain, depression, improves sleep and immunity which make mother to feel more energized and less fatigued. Massage is also a good option for postnatal mothers with fatigue who are not capable to do exercises. ${ }^{4}$

Post delivery massage is as important and beneficial as massage during pregnancy. Postnatal massage benefits include hormone regulation, better sleep, reduced swelling and improved breast feeding. ${ }^{5}$

Mustard oil is versatile oil that is both aromatic as well as soothing to the skin and can be used for therapeutic purpose. A massage with mustard oil helps to relieve rheumatic pain and improves blood circulation. Mustard oil is often a first choice for a post pregnancy massage because it is believed to be warming. 
Sometimes garlic is added to the mustard oil and the oil is slightly heated to give a warming effect. ${ }^{6}$

\section{RESEARCH STATEMENT}

A quasi-experimental study to assess the effectiveness of garlic scented mustard oil foot massage on fatigue after childbirth among postnatal mothers in selected hospitals of district Mohali, Punjab.

\section{OBJECTIVES}

1. To assess the pre-interventional fatigue after child birth among postnatal mothers in both experimental and control group.

2. To plan and conduct garlic scented mustard oil foot massage sessions among postnatal mothers in an experimental group.

3. To assess the post-interventional fatigue after childbirth among postnatal mothers in an experimental group.

4. To evaluate the effectiveness of garlic scented mustard oil foot massage on fatigue after childbirth among postnatal mothers.

5. To associate pre-interventional fatigue with selected socio-demographic variables.

OPERATIONAL DEFINITIONS

Effectiveness: It refers to the outcome of foot massage sessions with garlic scented mustard oil on fatigue assessed by self-structured postnatal fatigue assessment scale and visual analogue fatigue scale among postnatal mothers after child birth.

Garlic scented mustard oil: It is a homemade preparation in which 10 gram crushed garlic is mixed with $100 \mathrm{ml}$ of mustard oil and then the mixture is heated together for 5 minutes at medium flame. After cooling the mixture, oil is strained out and stored for use.

\section{Foot massage:}

Duration: 20 minutes massage once for two days (10 minutes each foot).

\section{Procedure:}

a) Wash hands and spread mackintosh with draw sheet under the feet.

b) Place the pillow under the calf muscles to ensure free movements of feet while massaging.

c) Pour small amount of oil on the palm of one hand and apply it gently using another hand along with to spread it evenly on feet.

d) Start the massage with one foot as described below:-
- Hold the foot with the fingers of both hands and rub the anterior aspect of the foot using thumbs of both hands in circular movements from toes to ankle and then repeat the circular movements back from ankle to toes.

- Hold the foot with one hand and with the other hand rub the anterior aspect of the foot with thumb using long strokes starting from big toe and move slowly towards ankle. Move the thumb back from ankle to big toe using long strokes. Repeat the step with index toe, middle toe, fourth toe and little toe.

- Hold the foot with the fingers of both hands and rub the sole of the foot using thumbs of both hands in circular movements from toes to heel and move back from heel to toes.

- Rub the lateral sides of foot with finger pads using long strokes from toe to ankle and back from ankle to toe.

e) Repeat the above mentioned steps with the other foot.

f) Remove excessive oil from the skin of both feet with the help of clean towel.

Fatigue: It refers to a state of physical and emotional exhaustion experienced by postnatal mothers after child birth, assessed through self-structured postnatal fatigue assessment scale and visual analogue fatigue scale.

Child birth: It refers to the process of giving birth to the baby.

Postnatal mother: It refers to primiparous woman within 24 hours of spontaneous or assisted vaginal delivery, excluding first 6 hours after delivery.

\section{HYPOTHESIS:}

$\mathbf{H}_{1}-$ The garlic scented mustard oil foot massage will be effective on fatigue after childbirth among postnatal mothers.

$\mathbf{H}_{2}-$ There will be a significant association between pre-interventional fatigue and selected sociodemographic variables.

METHODOLOGY

RESEARCH APPROACH: A quantitative research approach was adopted for the study.

RESEARCH DESIGN: Quasi-experimental non randomized control group research design was chosen. SETTING OF THE STUDY: Study was conducted in Civil hospital, phase VI, Mohali and Civil hospital, 
Kharar, Punjab. The reasons for selecting these hospitals were availability of subjects and ease in permission.

POPULATION: Target Population comprised of primiparous postnatal mothers admitted in selected hospitals of district Mohali, Punjab.

SAMPLE AND SAMPLING TECHNIQUE: The study sample consisted of 80 primiparous postnatal mothers who fulfilled the inclusion criteria. Among these 80 postnatal mothers, 40 were included in experimental group and 40 in control group. Non probability purposive sampling technique was used for the study.

\section{CRITERIA FOR SAMPLE SELECTION}

The inclusion and exclusion criteria used for the present study were as follows:

\section{Inclusion criteria:}

The study included primiparous postnatal mothers:

1. within 24 hours of spontaneous or assisted vaginal delivery.

2. willing to participate in the study.

3. available during data collection period.

4. able understand and communicate in Hindi/ Punjabi/English.

\section{Exclusion criteria:}

The study excluded primiparous postnatal mothers:

1. within first 6 hours after childbirth.

2. with presence of any diagnosed fatigue aggravating conditions during childbirth process such as anaemia.

3. who had delivered a high risk newborn.

4. with anatomical or physiological foot abnormality.

5. with pedal trauma.

6. who were allergic to garlic or mustard oil.

7. non cooperative during foot massage sessions.

\section{DEVELOPMENT OF TOOL}

To accomplish the objectives of the study, selfstructured postnatal fatigue assessment scale was used to assess fatigue among postnatal mothers. The tool consisted of three sections. Section I consisted of sociodemographic variables, section II consisted of selfstructured postnatal fatigue assessment scale and section III consisted of visual analogue fatigue scale.

Section I: Socio-demographic variables

This section consisted of 6 variables such as age (in years), type of family, educational status, working status, monthly income of family (in rupees) and dietary pattern.

Section II: Self-structured postnatal fatigue assessment scale was used with 3 point fatigue assessment rating scale. It consisted of 8 items to assess the severity of fatigue. A single tick $(\sqrt{ })$ on each item was marked by the researcher as per the symptom severity experienced and expressed by the participant.

Section III: Visual analogue fatigue scale

This was 10 point visual analogue fatigue assessment scale with point 0 on the one end of scale indicating no fatigue ascending to point 10 on the extreme opposite indicating severe fatigue. A tick was marked by the researcher on any point of this scale as expressed by the participant.

\section{RESULT}

SECTION-1: Frequency and percentage distribution according to socio-demographic variables.

According to the age, it was revealed that in the experimental group $48 \%$ were between the age group of 23-26 years whereas, in the control group 43\% were between the age group of 23-26 years.

According to the type of family, it was revealed that in the experimental group 58\% belongs to nuclear family whereas, in the control group $60 \%$ belongs to nuclear family.

Distribution of subjects in relation to their educational status revealed that $38 \%$ had attained education up to secondary level in experimental group whereas, in the control group 33\% had received education up to secondary level.

The working status of experimental group revealed that $58 \%$ were homemaker whereas, in the control group $40 \%$ of mothers were homemaker.

In relation to their monthly income revealed that in the experimental group 38\% had income between 10,00120,000 rupees whereas, in the control group $53 \%$ of postnatal mothers had income less than 10,000 rupees.

The dietary pattern of experimental group revealed that $38 \%$ of subjects were vegetarian whereas, in the control group $55 \%$ of postnatal mothers were vegetarian.

SECTION II (A): Effectiveness of garlic scented mustard oil foot massage on fatigue among postnatal mothers through postnatal fatigue assessment scale. 
$\mathbf{N}=\mathbf{8 0}$ hypotheses $\mathrm{H}_{2}$ was rejected in case of selected socio-

\begin{tabular}{|c|c|c|c|c|c|c|c|}
\hline & $\begin{array}{c}\text { Experimental } \\
\text { group } \\
\mathbf{n}=\mathbf{4 0}\end{array}$ & \multicolumn{2}{c|}{$\begin{array}{c}\text { Control } \\
\text { group } \\
\mathbf{n}=\mathbf{4 0}\end{array}$} & \multicolumn{3}{|c|}{ Unpaired t-test } \\
\hline & Mean & SD & Mean & SD & df & $\mathbf{t}$ & p-value \\
\hline Pretest & 11.30 & 0.883 & 11.100 & 1.105 & 78 & 0.894 & $0.3738^{\mathrm{NS}}$ \\
\hline Posttest & 5.03 & 0.620 & 10.75 & 1.878 & 78 & 18.312 & $0.000^{*}$ \\
\hline
\end{tabular}

NS=Not significant

* significant $\mathbf{p}<0.05$

SECTION II (B): Effectiveness of garlic scented mustard oil foot massage on fatigue among postnatal mothers through visual analogue fatigue scores.

$\mathbf{N}=\mathbf{8 0}$

\begin{tabular}{|c|c|c|c|c|c|c|c|}
\hline & $\begin{array}{c}\text { Experimental } \\
\text { group } \\
\text { n=40 }\end{array}$ & \multicolumn{2}{c|}{$\begin{array}{c}\text { Control } \\
\text { group } \\
\text { n=40 }\end{array}$} & \multicolumn{3}{|c|}{ Unpaired t-test } \\
\hline & Mean & SD & Mean & SD & df & t & p-value \\
\hline Pretest & 7.38 & 1.213 & 7.700 & 1.137 & 78 & 1.236 & $0.2200^{\mathrm{NS}}$ \\
\hline Posttest & 2.93 & 0.917 & 7.50 & 1.219 & 78 & 18.966 & $0.000^{*}$ \\
\hline
\end{tabular}

NS $=$ Not significant

* significant $\mathbf{p}<0.05$

SECTION III (A): Association of pre-interventional postnatal fatigue assessment scores with selected socio-demographic variables in experimental group and control group.

In experimental experiment, the chi-square revealed that the selected socio-demographic variables except dietary pattern, do not have impact on fatigue after childbirth. Hence, research hypotheses $\mathrm{H} 2$ was rejected in case of selected socio-demographic variables except dietary pattern.

In control group, the chi-square showed that the selected socio-demographic variables do not have impact on fatigue after childbirth. Hence, research hypotheses $\mathrm{H}_{2}$ was rejected in case of a sociodemographic variables.

SECTION III (B): Association of pre-interventional visual analogue fatigue scores with selected sociodemographic variables in experimental group and control group.

In experimental group, the chi-square showed that the selected socio-demographic variables except dietary pattern, do not have impact on fatigue after childbirth. Hence, research hypotheses $\mathrm{H}_{2}$ was rejected in case of selected socio-demographic variables except dietary pattern.

In control group, the chi-square showed that the selected socio-demographic variables except educational status and working status, do not have impact on fatigue after childbirth. Hence, research working status.

\section{IMPLICATIONS OF THE STUDY}

Nursing practice: Foot massage can be made to practice as a routine nursing midwifery care after child birth to reduce the level of fatigue along with other measures of comfort after child birth nursing. This intervention is cost-effective, safe and easy to practice.

Nursing Education: The nurse educator should encourage the students to learn about the assessment of fatigue after child birth and its consequences on mothers and newborns health and also the remedial measures to reduce the fatigue after child birth.

Nursing Administration: The nurse administrator can organize conferences and in-service education programmes on various non pharmacological measures and therapies in reduction of fatigue after child birth

Nursing Research: The nurse researcher should motivate the clinical nurse to do further research studies on effectiveness of foot massage on other postnatal discomfort like leg pain, backache and postnatal blues etc.

\section{REFERENCES}

1. Dennis C, Fung K, Grigoriadis S, Robinson G, Romans S, Ross L. Traditional Postpartum Practices and Rituals: A Qualitative Systematic Review. Women's Health. 2007;3(4):487-502

2. Asazawa K, Kato Y, Yamaguchi A, Inoue A. The Effect of Aromatherapy Treatment on Fatigue and Relaxation for Mothers during the Early Puerperal Period in Japan: A Pilot Study. Int J Community Based Nurs Midwifery. 2017;5(4):365-375.

3. Shimada M, Sugimoto M, Agata T, Nitta N, Seki K, Ohashi K et al. Nation- wide survey on maternal anxiety in one- month postpartum, needs for childrearing supports and environment at 5 years after healthy parents and children 21 programs. The Journal of Child Health. 2006;65:752-62.

4. Massage and chronic fatigue [Internet]. Pacific college of oriental medicine. 2019 [cited 3 March 2019]. Available from: http://www.pacificcollege. edu/news/blog/2014/11/23/massage-andchronicfatigue

5. Postpartum massage [Internet]. 2019 [cited 3 March 2019]. Available from:https://ameri canpregnancy.org/first-year-of-life/post partum massage/

6. Health benefits of mustard oil [Internet]. 2016 [cited 13 March 2019]. Available from:http://www. dhanyy.hu bpages.com 